\title{
УДК 004.89
}

СЕМАНТИЧЕСКИЕ ТЕХНОЛОГИИ УПРАВЛЕНИЯ ЗНАНИЯМИ ДЛЯ

ПОДДЕРЖКИ СОВМЕСТНЫХ ЭНЕРГЕТИЧЕСКИХ И СОЦИО-ЭКОЛОГИЧЕСКИХ ИССЛЕДОВАНИЙ

\section{Ворожцова Татьяна Николаевна}

к.т.н., вед. инж. отдела «Системы искусственного интеллекта в энергетике»,

$$
\text { e-mail: tnn@isem.irk.ru, }
$$

\section{Пестерев Дмитрий Вячеславович}

инж. отдела «Системы искусственного интеллекта в энергетике», e-mail: pesterev.dmitriy@gmail.com,

\section{Ан Глеб Владимирович}

асп. отдела «Системы искусственного интеллекта в энергетике», e-mail: godblessya95@gmail.com,

Институт систем энергетики им. Л.А. Мелентьева СО РАН, 664130 г. Иркутск, ул. Лермонтова 130.

Аннотация. В статье рассматриваются возможности применения семантических технологий при разработке интеллектуальной информационной системы для поддержки энергетических и социо-экологических исследований. Использование предлагаемых технологий для управления знаниями дает возможность интеграции исследований разных предметных областей. К семантическим технологиям авторы относят традиционное онтологическое представление знаний и когнитивное моделирование, позволяющее формализовать процессы принятия экспертных решений. Использование онтологий предлагается для описания знаний предметных областей междисциплинарных исследований (энергетических и социо-экологических), связанных с оценкой влияния энергетики на качество жизни. Онтологии дают возможность согласовать терминологию между исследователями и используются для описания и структурирования знаний. Когнитивное моделирование применяется для поддержки исследователей в процессе принятия решений, выявления и описания причинно-следственных связей между основными понятиями предметной области, называемыми концептами, их наглядного представления в процессе описания возможных ситуаций. В работе описываются компоненты онтологического пространства знаний для выполнения совместных энергетических и социо-экологических исследований влияния энергетики на экологию и качество жизни. Приводятся примеры онтологии, когнитивной карты и варианта разработанной модели данных для проектирования базы данных исследований.

Ключевые слова: семантическое моделирование, когнитивное моделирование, онтология, онтологическое пространство, когнитивная карта, качество жизни, поддержка принятия решений, база данных.

Цитирование: Ворожцова Т. Н., Пестерев Д.В., Ан Г.В. Семантические технологии управления знаниями для поддержки совместных энергетических и социо-экологических исследований //Информационные и математические технологии в науке и управлении. 2020. № 4 (20). С.143157. DOI: $10.38028 /$ ESI.2020.20.4.013 
Введение. В ИСЭМ СО РАН выполняется проект «Методы построения онтологического пространства знаний для интеллектуальной поддержки принятия решений в энергетике и экологии с учетом качества жизни», поддержанный грантом РФФИ № 20-0700195. Актуальность данной работы обусловлена необходимостью обеспечить поддержку принятия согласованных решений в области энергетики с учетом ее влияния на экологию и качество жизни. Для этого разрабатываются и используются такие типы семантического моделирования, как когнитивное и онтологическое моделирование. В рамках данного проекта предполагается разработка интеллектуальной информационной системы (ИИС), которая интегрирует сложную информационную базу для выполнения исследований, методы математического и семантического моделирования, инструментальные средства оценки влияния энергетики на экологию и качество жизни. Наличие и функционирование объектов энергетики на той или иной территории влияет на качество жизни населения, так как, с одной стороны, обеспечивает потребности в необходимой тепловой и электрической энергии, а с другой стороны, является одним из источников загрязнений окружающей среды. Разрабатывается единое онтологическое пространство знаний, обеспечивающее интеграцию исследований предметных областей энергетики и экологии. Система онтологий обеспечивает информационную поддержку исследований путем интеграции необходимых данных, проектирования и разработки баз данных на основе онтологического описания. Использование методов онтологического и когнитивного моделирования и поддерживающих их инструментальных средств предлагается для согласования энергетических и социоэкологических исследований, а именно, для научного обоснования принятия решений в энергетике с учетом ее влияния на экологию и качество жизни.

Возможности и цели онтологического моделирования. Для интеллектуальной поддержки междисциплинарных исследований с учетом пересечения знаний разных предметных областей предлагается использовать семантические технологии для описания и моделирования знаний. Моделирование знаний о предметных областях - главное направление и базовая парадигма искусственного интеллекта. В настоящее время онтологический подход является основным в разработке и внедрении информационных систем и систем управления знаниями [7,10,11]. Онтологическое моделирование - одно из ведущих направлений семантического моделирования [21, 23]. Вопросы применения онтологического моделирования в исследованиях энергетики рассматривались в работах $[5,15,16]$. При этом авторами используются онтологический инжиниринг, как основной метод работы со знаниями, и фрактальный подход для структурирования знаний.

Онтологический инжиниринг - это процесс проектирования и разработки онтологий на основе структурного анализа предметной области [7].

Знания о предметной области представляют собой совокупность сведений об объектах этой предметной области, свойствах этих объектов, отношениях между объектами, а также о процессах и ситуациях, происходящих в данной предметной области. Процесс онтологического инжиниринга включает следующие шаги:

- выделение базовых понятий данной предметной области - концептов,

- построение связей или отношений между концептами,

- выстраивание иерархии концептов,

- описание свойств выбранных понятий. 
Основным преимуществом онтологического инжиниринга является то, что при этом достигаются:

- $\quad$ системность - онтология представляет целостный взгляд на предметную область;

- единообразие - материал представляется в единой форме для лучшего восприятия;

- $\quad$ научность - построение онтологии позволяет восстановить недостающие логические связи во всей их полноте.

Возможности применения предложенного Массель Л.В. фрактального подхода к структурированию знаний и построению онтологического пространства описаны в [17]. При использовании фрактального подхода предполагается, что знания об изучаемом объекте представляются в виде совокупности (множества) слоев, каждый из которых характеризует определенный аспект данных, информации или знаний об объекте.

Онтологические модели дают следующие возможности работы со знаниями:

- являются средством представления знаний;

- обеспечивают работу со смыслом информации;

- допускают автоматизированную обработку;

- обеспечивают интеграцию приложений;

- обеспечивают возможность логических выводов.

В данной работе система онтологических моделей предназначена для:

- согласования исследований разных предметных областей (энергетики, экологии, качества жизни) в соответствии с целями проекта,

- согласования понятий этих предметных областей,

- обеспечения доступности и восприятия больших объемов сложной и/или слабоструктурированной информации,

- описания структуры и компонентов ИИС.

Компоненты онтологического пространства знаний. Система онтологий разрабатываемого онтологического пространства знаний для исследований обеспечивает представление знаний для взаимосвязанных исследований энергетики, экологии и качества жизни.

Онтологическое пространство знаний базируется на фрактальном подходе, предполагающем введение нескольких слоев - метауровней, и их дальнейшее расслоение, предусматривающее все большую степень детализации на каждом следующем уровне.

Метаонтологии включают базовые понятия соответствующих предметных областей энергетики, экологии и качества жизни, имеющие отношение к совместным исследованиям. Они используются как основа для описания базовых компонентов и структуры ИИС, а также для разработки ее интерфейса.

Выделены следующие уровни (слои) онтологий:

- Онтологическое описание предметных областей исследований - энергетика, экология, качество жизни.

- Онтологии, описывающие используемые данные и информацию, онтологии баз данных (БД).

- Онтологическое описание баз знаний, содержащих не только описание классов и экземпляров, их свойств и взаимосвязей, но и набор правил, обеспечивающих возможность логического вывода. 
На рисунке 1 приведена метаонтология, объединяющая базовые понятия предметных областей и отражающая основные разделы исследований - энергетика, экология и качество жизни. Представленные понятия являются базовыми классами системы онтологий. Компоненты ИИС соответствуют этим разделам. Раздел «Энергетика» содержит описание объектов энергетики и их свойств, от которых зависит антропогенное влияние на природную среду и качество жизни. В разделе «Экология» описываются антропогенные факторы, элементы природной среды и методики оценки антропогенного влияния. Понятие «Качество жизни» - это совокупная характеристика уровня и объективных и субъективных условий жизни населения, определяющих физическое, ментальное, социально-культурное развитие человека, группы или сообщества людей [4]. По определению Всемирной организации здравоохранения (ВО3) качество жизни - это восприятие индивидами их положения в жизни в контексте культуры и системы ценностей, в которых они живут, в соответствии с целями, ожиданиями, нормами и потребностями. Соответственно раздел «Качество жизни» включает описание индикаторов качества жизни, факторов влияния и методов анализа этого влияния. Раздел «Задача» содержит описание решаемых в данных исследованиях задач. Раздел «Методология», как компонент ИИС, включает совокупность методических подходов в исследованиях, методов и алгоритмов, используемых для оценки влияния объектов энергетики на экологию и качество жизни.

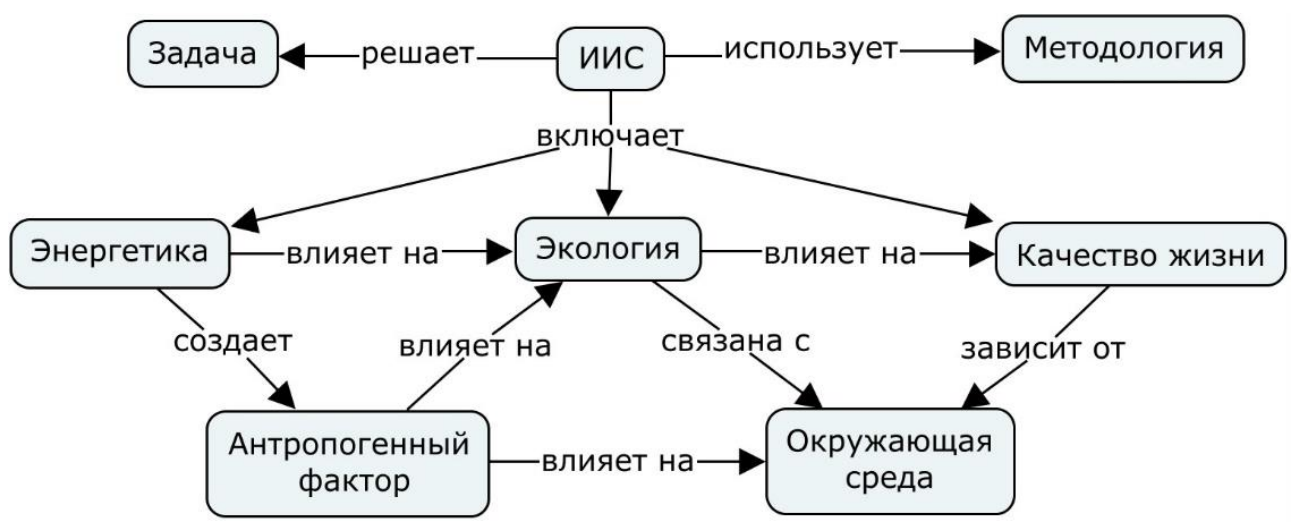

Рис. 1. Метаонтология предметной области исследований

Одним из важных концептов представленной метаонтологии является «Антропогенный фактор» - причина антропогенного воздействия на окружающую среду, обусловленная процессом и условиями функционирования объекта, его характерными особенностями. Применительно к энергетическим объектам под антропогенными факторами понимаются эмиссии, отходы, излучение, шум, вибрация, радиация и т.п. Антропогенный фактор зависит от типа и вида энергоресурса и типа технологий энергетического объекта. Через показатели антропогенного влияния на окружающую среду экспертно оценивается степень отрицательного влияния на качество жизни населения.

Когнитивное моделирование и его применение в исследованиях. Когнитивный анализ и моделирование являются современными интеллектуальными технологиями и используются для исследования слабоформализованных и слабоструктурированных систем, к которым относятся экономические, социальные, экологические системы $[2,20]$.

В настоящее время применение когнитивного моделирования развивается в направлении моделирования и анализа ситуаций и принятия решений. За рубежом наиболее 
ярко это отражено в работах П. Грумпоса и Х. Стайлиоса [22]. В нашей стране это научная школа Кулинича $[12,13]$, а также работы сотрудников Института Проблем Управления РАН [1] и др. В ИСЭМ СО РАН предложено использовать когнитивное моделирование как инструмент для исследования энергетической безопасности (ЭБ) для изучения связей между основными составляющими проблемы ЭБ и получения выводов об их взаимовлиянии $[14,16]$.

В основе когнитивного анализа лежит познавательно-целевая (когнитивная) структуризация знаний об объекте и внешней для него среды. Когнитивная структуризация это выявление наиболее существенных факторов, влияющих на ситуацию, и причинноследственных связей между ними. Методология когнитивного моделирования основана на моделировании субъективных представлений экспертов о ситуации. Знания экспертов графически представляются в виде когнитивной карты.

В работе предлагается использовать когнитивное моделирование для анализа факторов взаимовлияния энергетики и экологии на качество жизни населения. Использование онтологий в данном случае необходимо для выявления этих факторов и формирования списка концептов. В онтологическом пространстве, организуемом для изучаемого междисциплинарного взаимодействия, существуют факторы одной предметной области (ПрО), которые могут как положительно, так и отрицательно влиять на факторы другой (ПрО). Например, факторы из области энергетики оказывают как положительное влияние на качество жизни (использование электроэнергии, использование тепловой энергии), так и отрицательное (загрязнение окружающей среды, шумовое воздействие и пр.). Поэтому основная задача когнитивного моделирования - установить взаимовлияние факторов в той или иной конкретной ситуации. Другая задача - визуализация зависимостей между основными факторами исследуемой ПрО.

Для построения когнитивной карты выполняется анализ предметной области, выделяются основные факторы (концепты), которые имеют значения для исследования. Если уже существует онтология предметной области, то концепты выделяются непосредственно на основе этой онтологии. После этого этапа между концептами устанавливаются связи и их характер. В самом простом случае связи могут быть либо положительными, либо отрицательными. В более сложном случае к знакам добавляются весовые коэффициенты, получаемые методом экспертных оценок. При анализе конкретной ситуации когнитивная карта строится либо заново, либо выбирается из числа уже существующих, при условии, что содержит необходимые концепты предметной области.

На рисунке 2 показан пример когнитивной карты, которая описывает основные факторы ситуации «Обеспечение населения тепловой энергией» с позиции основного фактора «Качество жизни». 


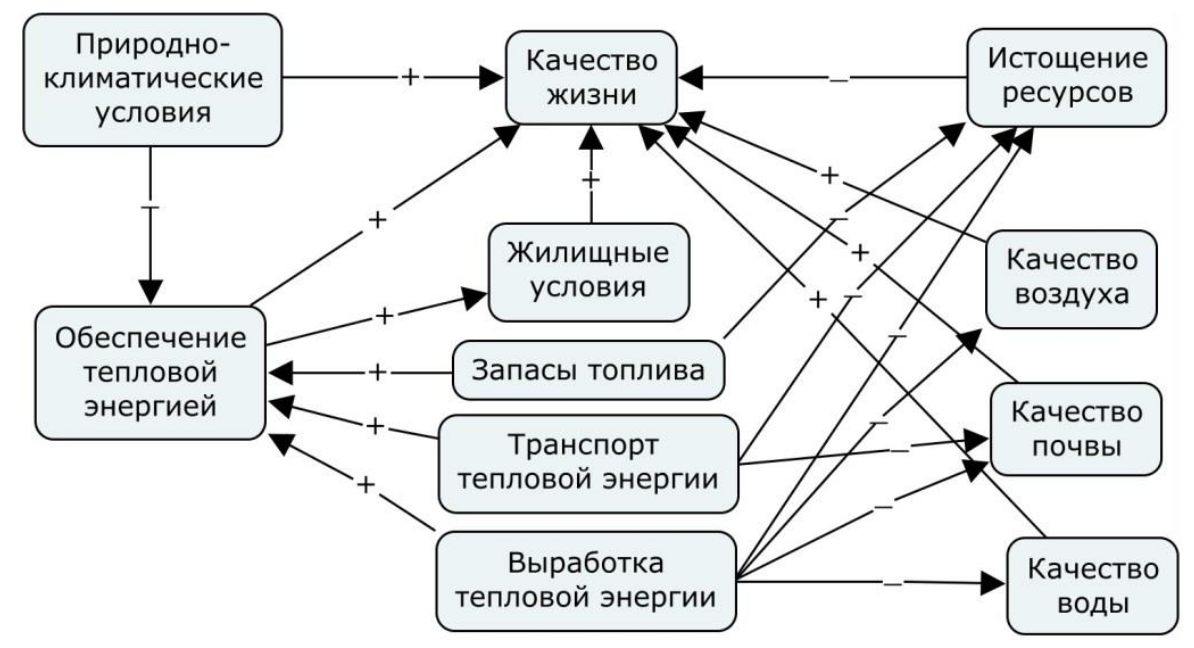

Рис. 2. Когнитивная карта «Обеспечение населения тепловой энергией».

На этой карте видно влияние факторов, оказываемое на целевой фактор «Качество жизни». Это влияние как положительно, так и отрицательно. Чтобы точно установить суммарное влияние, потребуется введение весовых коэффициентов, которые определяются методом экспертных оценок.

Онтологии и модель данных. Создание информационных систем с использованием онтологического подхода обеспечивает значительное преимущество с точки зрения корректной подготовки информационных компонентов [19]. Использование семантических и онтологических стандартов помогает наладить обмен и сопоставление данных, выявление коллизий и согласование противоречий, также онтологическая модель является удобным базисом для разработки модели данных [6].

Как правило, проектирование базы данных состоит из последовательных шагов, результатом выполнения которых является создание готовых моделей данных [8]. Процесс проектирования БД состоит из следующих этапов:

1) проектирование на уровне концепций - определение общей концепции БД с учетом включения важнейших сущностей (таблиц) и связей между ними, но не зависящей от типа СУБД (иерархической, сетевой, реляционной и т.д.) и физической реализации (целевой СУБД); при использовании семантического подхода создается онтологическая модель;

2) логическое проектирование - развитие концептуальной модели данных с учетом типа выбранной СУБД (иерархической, сетевой, реляционной и т.д.);

3) проектирование физической БД - конкретизация логической модели данных с учетом выбранной целевой СУБД.

Разрабатываемый программный компонент преобразования онтологии в модель данных является важнейшим компонентом реализации идеи создания интеллектуальной информационной системы на основе онтологий. При анализе онтологических моделей выделяют важные для предметной области концепты и отношения, которые в конечном итоге используются для построения модели типа «сущность-связь» [8]. Для корректного построения модели данных при анализе онтологии выделяют следующие элементы [3]:

- сущности - выделенный набор (класс) однотипных концептов, обобщенных под каким-то одним общим концептом; 
- $\quad$ связи - отношения между концептами (связи), кроме случаев, когда связь является «обобщающей» (такие связи правильнее использовать для построения сущности, т.е. таблицы);

- свойства (атрибуты) - дополнительные характеристики концепта, которые в онтологической модели детализируются с помощью других концептов (концептовхарактеристик). Основная особенность концептов-характеристик в том, что они предполагают некоторый описательный вид, вариативны - могут принимать различные строковые значения.

В качестве примера преобразования онтологической модели в реляционную базу данных (предполагается, что этап концептуального проектирования выполняется экспертом предметной области) выбрана онтология видов энергоисточников (рисунок 3).

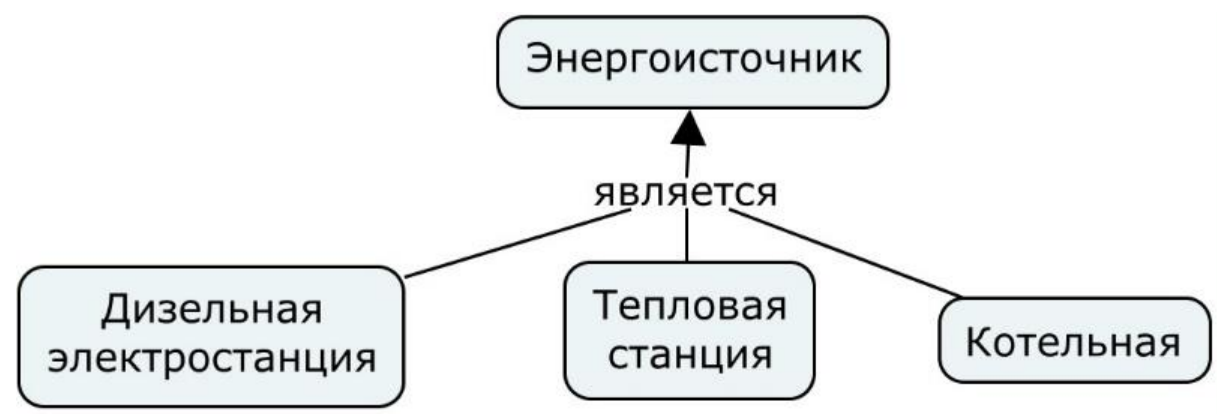

Рис. 3. Онтология «Энергоисточники»

При анализе онтологии можно сделать вывод, что концепт «Энергоисточник» является сущностью (обобщением) объектов «Дизельная э/станция», «Тепловая э/станция», «Котельная». Преобразовав исходную онтологическую модель, получим инфологическую модель данных, представленную на рисунке 4.

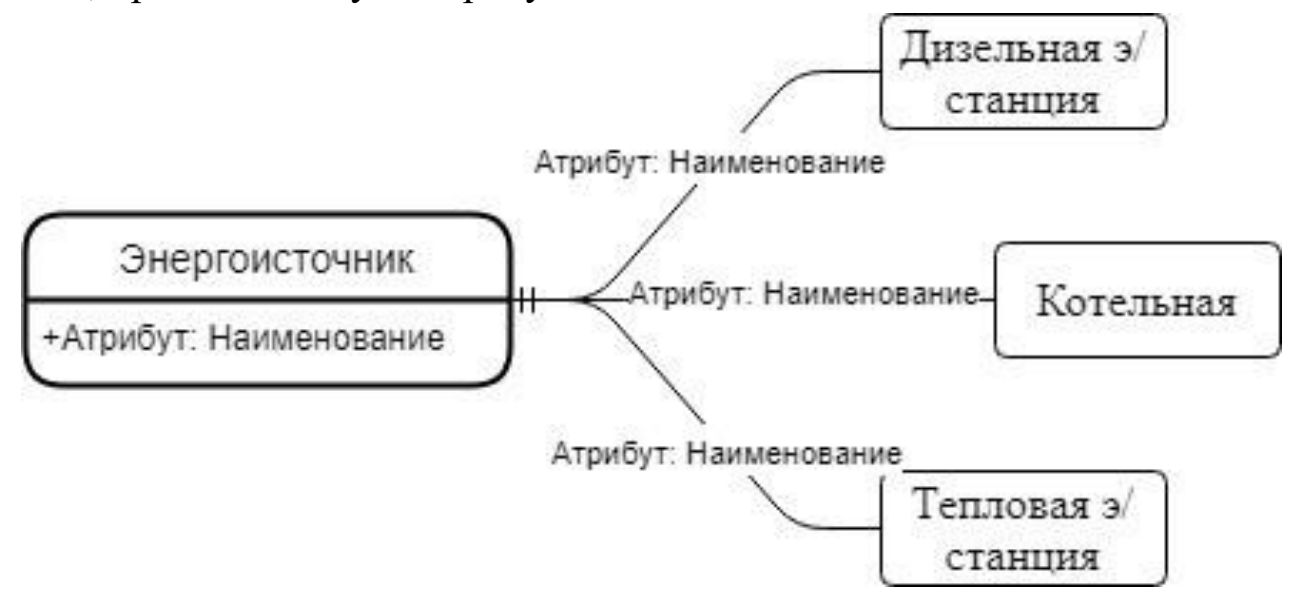

Рис. 4. Инфологическая модель данных

В спроектированной инфологической модели можно выделить следующие элементы: сущность (отношение) «Энергоисточник» и элементы (значения) с наименованиями дизельная/котельная/тепловая станция, которые являются частью спроектированного отношения (данными будущей таблицы) и будут записаны в физическую таблицу под атрибутом «Наименование». Так как в исходной онтологической модели отсутствовали какиелибо дополнительные концепты-характеристики, то нет необходимости в расширении полученной сущности-таблицы. 
Завершающий этап проектирования БД - это создание физической модели данных. Физическое проектирование осуществляется путем создания новой базы с именем, соответствующем названию онтологии, в нашем примере БД получит имя «Описание видов энергоисточников». При преобразовании сущности «Энергоисточник» в физическую таблицу БД необходимо сделать уникальной каждую запись в таблице с помощью добавления уникального атрибута таблицы (первичного ключа) id_station. На рисунке 5 показана спроектированная таблица данных.

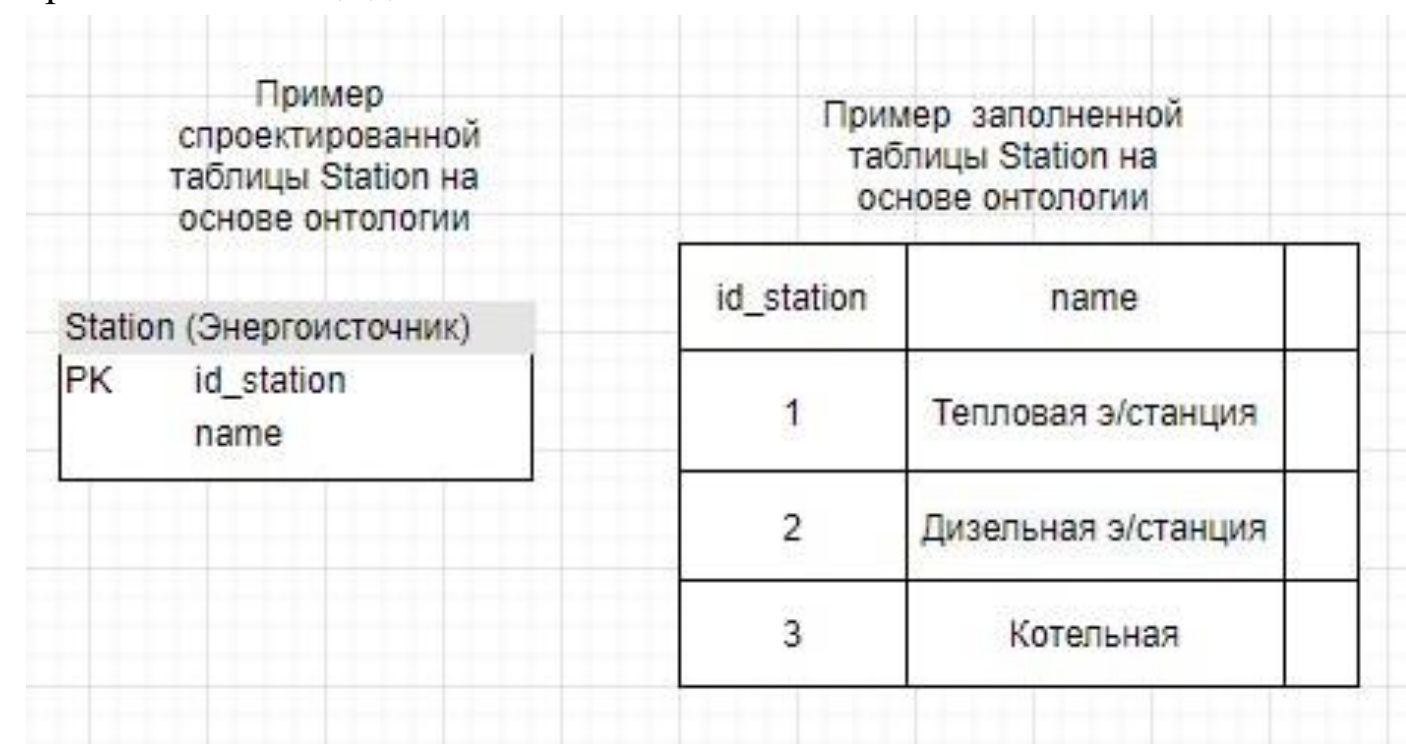

Рис. 5. Пример спроектированной в БД таблицы «Энергоисточник»

Таким образом, выполнив все этапы проектирования (концептуальное, инфологическое, физическое), получаем готовую базу данных, которая будет использована при реализации интеллектуальной информационной системы.

На основании описанных особенностей проектирования БД авторами построена блоксхема алгоритма преобразования онтологии в модель данных, показанная на рисунке 6 .

На рисунке 7 представлен полный вид физической модели данных для хранения информации по объекту энергетики - котельной. Поскольку онтология использует атрибуты естественного языка, то для ее согласования с моделью данных необходимо ограничить набор допустимых связей между концептами («имеет», «является», «измеряется в», «может принимать значение», «состоит из»).

В дальнейшем на основе построенной модели данных будет сформирована информационная система, которая будет выполнять роль информационной поддержки экспертам при принятии решений в энергетике и экологии.

Заключение. В работе описан пример применения семантических технологий управления знаниями [18] для выполнения энергетических и социо-экологических исследований. Для этого разрабатывается интеллектуальная информационная система, которая интегрирует сложную методологическую и информационную базу междисциплинарных исследований. Для обеспечения поддержки принятия согласованных решений в области энергетики с учетом ее влияния на экологию используются методы семантического моделирования, включающие онтологическое и когнитивное моделирование. Разрабатывается единое онтологическое пространство знаний, обеспечивающее интеграцию исследований ПО энергетики и экологии. 


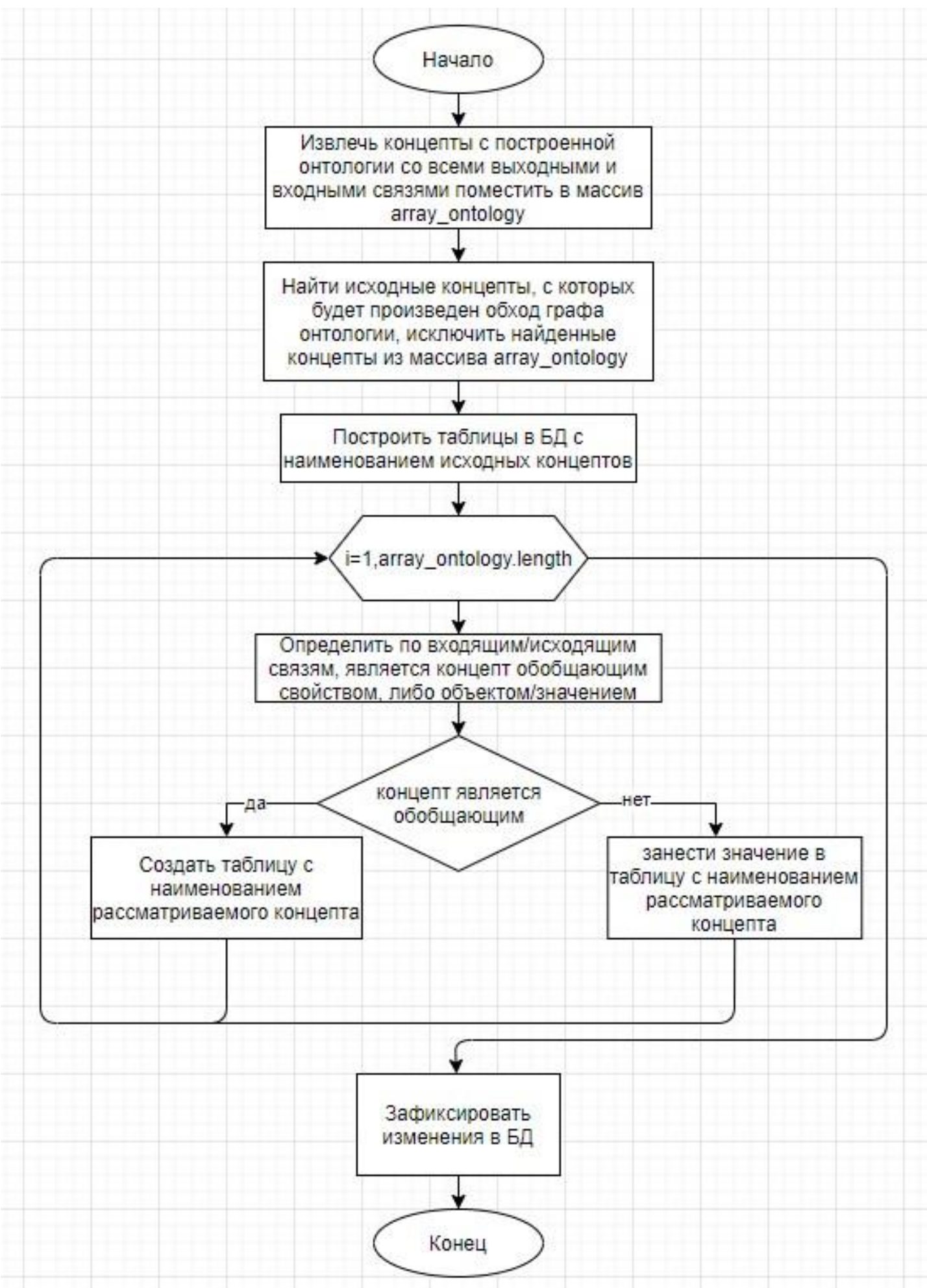

Рис. 6. Алгоритм преобразования онтологии в модель данных

Разрабатываемая система онтологий обеспечивает информационную поддержку исследований путем интеграции необходимых данных, проектирования и разработки баз данных на основе онтологического описания. Онтологии используются для выявления и согласования базовых понятий разных предметных областей, имеющих отношение к совместным исследованиям, установления взаимосвязей между ними, а также для структурного представления знаний. 


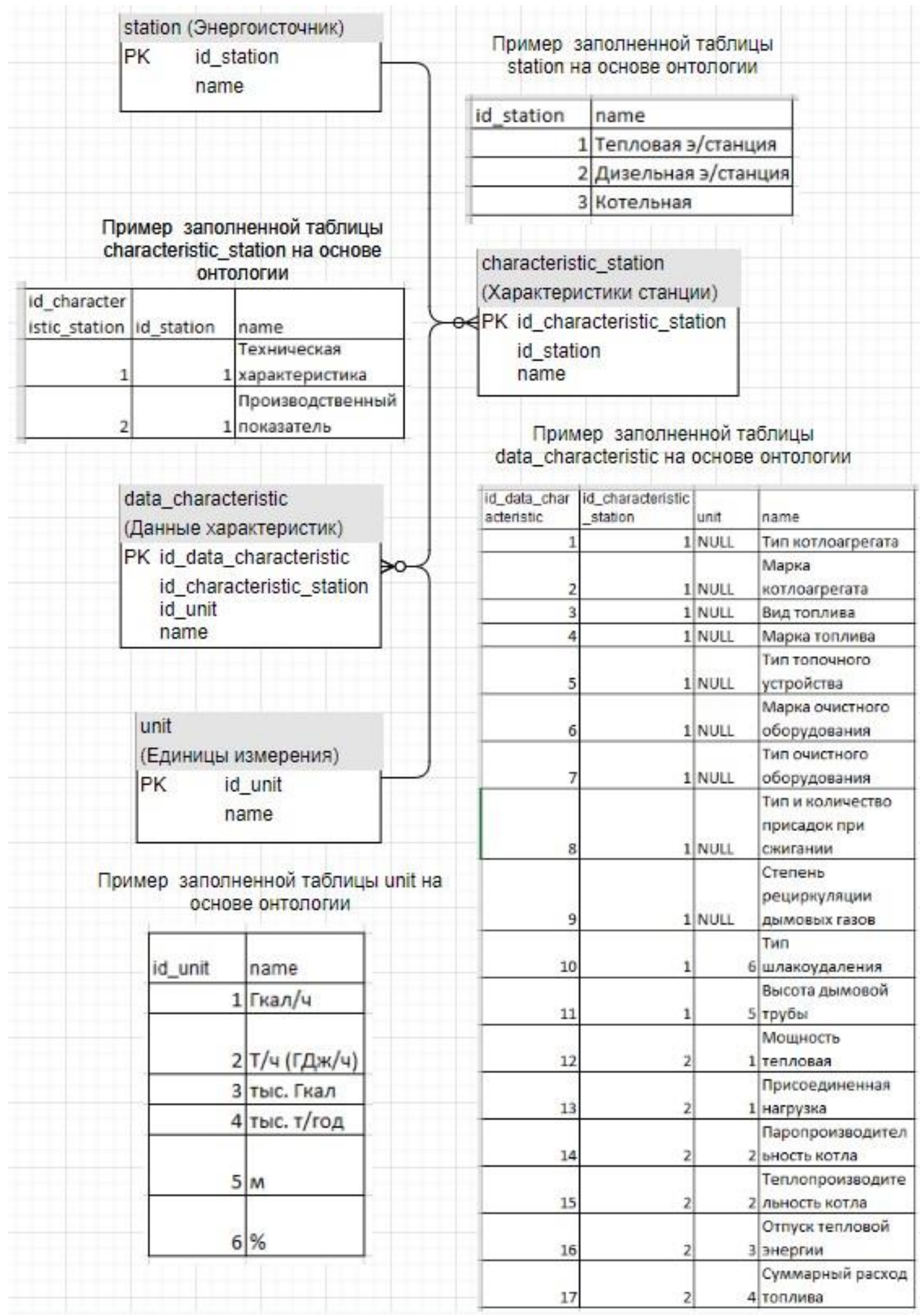

Рис. 7. Физическая модель данных котельной

Когнитивное моделирование применяется для анализа и моделирования ситуаций и принятия согласованных решений в энергетических исследованиях с учетом влияния энергетики на экологию и качество жизни. Когнитивные модели используются для выявления причинно-следственных связей между концептами, их наглядного представления в процессе описания возможных ситуаций и принятия решений. 
Благодарности. Результаты получены в рамах выполнения проекта по госзаданию ИСЭМ СО РАН АААА-А17-117030310444-2 (проект №349-2016-0005) и при частичной финансовой поддержке грантов РФФИ № 19-07-00351, № 20-07-00195.

\section{СПИСОК ЛИТЕРАТУРЫ}

1. Авдеева 3.К., Горелова Г.В., Коврига С.В. Когнитивные исследования качества жизни населения города // Труды Седьмого Международного форума по когнитивному моделированию. В 3-х частях: Труды Творческой школы, Труды XX Международной конференции, Труды VII Международной конференции. 2019. С. 205-214.

2. Авдеева 3.К., Коврига С.В., Макаренко Д.И. Когнитивное моделирование для решения задач управления слабоструктурированными системами (ситуациями) // Управление большими системами. 2007. №16. С. 26-39.

3. Баркер P. CASE*Method. Моделирование взаимосвязей между сущностями / М.. 1992. $233 \mathrm{c}$.

4. Большая российская энциклопедия, Режим доступа: https://bigenc.ru/vocabulary (дата обращения 8.10.2020).

5. Ворожцова Т.Н. Онтологическая модель пространства знаний для ситуационного управления в энергетике // Труды XX Байкальской Всероссийской конференции «Информационные и математические технологии в науке и управлении». Т.3 Иркутск: ИСЭМ СО РАН. 2015. С. 85-88.

6. Ворожцова Т.Н., Макагонова Н.Н., Массель Л.В. Онтологический подход к проектированию базы данных для оценки влияния энергетики на окружающую среду //Информационные и математические технологии в науке и управлении. 2019. № 3 (15). С. 31-41. DOI: 10.25729/2413-0133-2019-3-03.

7. Гаврилова Т. А., Кудрявцев Д. В., Муромцев Д. И. Инженерия знаний. Модели и методы. Санкт-Петербург. 324. (2016)

8. Дейт К. Дж. Введение в системы баз данных. 8-е издание. М.: Издательский дом "Вильямс". 2005. 1328 с.

9. Загорулько Ю. А., Боровикова О. И., Загорулько Г. Б. Роль Онтологии в Технологии Построения Тематических Научных Интернет-ресурсов // Всероссийская конференция с международным участием "Знания - Онтологии - Теории" (ЗОНТ-2015). - Новосибирск, Институт математики им. С.Л. Соболева СО РАН. - 2015. - С. 101- 110.

10. Загорулько Ю. А., Загорулько Г. Б., Онтологии и их практическое применение в системах, основанных на знаниях // Всероссийская конференция с международным участием "Знания - Онтологии - Теории" (ЗОНТ-2011). Новосибирск. Институт математики им. С.Л. Соболева СО РАН. 2011. Том 1.

11. Когаловский М.Р., Калиниченко Л.А. Концептуальное и онтологическое моделирование в информационных системах. Программирование. 2009. Т. 35. № 5. С. 3-25. 12. Кузнецов О.П., Кулинич А.А., Марковский А.В. Анализ влияний при управлении слабоструктурированными ситуациями на основе когнитивных карт // Человеческий фактор в управлении. М.: КомКнига. 2006. С. 313-344. 
13. Кулинич А.А. Семиотические когнитивные карты. Ч. 1. Когнитивный и семиотический подходы в информатике и управлении // Проблемы управления. 2016. № 1. C. 2-10.

14. Массель А.Г. Когнитивное моделирование в исследованиях проблем энергетической безопасности: применения и перспективы развития. // Труды Международного Конгресса по интеллектуальным системам и информационным технологиям «IS\&IT'14». М.: Физматлит. 2014. Т.2. С. 153-158.

15. Массель Л.В., Ворожцова Т.Н., Копайгородский А.Н., Макагонова Н.Н., Скрипкин С.К. Применение онтологий в исследованиях и поддержке принятия решений в энергетике //Всероссийская конференция с международным участием «Знания - Онтологии - Теории» (ЗОНТ-13). Новосибирск: ИМ СО РАН. Том 2. С. 29-38.

16. Массель Л.В. Применение онтологического, когнитивного и событийного моделирования для анализа развития и последствий чрезвычайных ситуаций в энергетике // Проблемы безопасности и чрезвычайных ситуаций. 2010. № 2. С.34-43.

17. Массель Л. В. Фрактальный подход к структурированию знаний и примеры его применения // Онтология проектирования. 2016. №2 (20). С. 149-161.

18. Тузовский А.Ф., Чириков С.В., Ямпольский В.З. Системы управления знаниями (методы и технологии). Томск: Изд-во НТЛ. 2005. 260 с.

19. Черников Б.В. Лексикологический синтез документов в комплексах информационных систем // M: «Форум». 2017. 336 с.

20. Axelrod R. The Structure of Decision: Cognitive Maps of Political Elites. Princeton. University Press. 1976

21. Gruber T.R. Towards principles for the design of ontologies used for knowledge sharing // International Journal of Human-Computer Studies. 1995. V. 43. (5/6). 907 - 928.

22. Groumpos P., Stylios C. Modelling supervisory control systems using fuzzy cognitive maps. Chaos. Solitons \& Fractals. 2000.Vol. 11(1-3). Pp.329-336.

23. Guarino N., Formal Ontology and Information Systems // Proceedings of International Conference of Formal Ontology and Information Systems (FOIS'98). Amsterdam: IOS Press. 1998. Pp. $3-15$. 


\title{
UDK 004.89
}

\section{SEMANTIC TECHNOLOGIES FOR KNOWLEDGE MANAGEMENT TO SUPPORT JOINT ENERGY AND SOCIO-ECOLOGICAL RESEARCH}

\author{
Tatiana N.Vorozhtsova \\ PhD, Leading engineer of Department “Artificial Intelligence Systems in Energy Sector" \\ e-mail: tnn@isem.irk.ru
}

Dmitriy.V.Pesterev

Engineer of Department “Artificial Intelligence Systems in Energy Sector” e-mail: pesterev.dmitriy@gmail.com

Gleb.V.An

Engineer of Department “Artificial Intelligence Systems in Energy Sector”

Melentiev Energy Systems Institute SB RAS, Irkutsk, Russia

e-mail: godblessya95@gmail.com

\begin{abstract}
The article discusses the possibilities of using semantic technologies in the development of an intelligent information system to support energy and socioenvironmental research. The use of the proposed knowledge management technology makes it possible to integrate research in different subject areas. The authors refer to the semantic technologies as the traditional ontological representation of knowledge and the cognitive approach, which allows formalizing the processes of making expert decisions. The use of ontologies is proposed to describe the knowledge of subject areas of interdisciplinary energy and socio-ecological research related to assessing the impact of energy on the quality of life. Ontologies make it possible to agree on terminology between researchers and are used to describe and structure knowledge and required information. Cognitive modeling is used to support researchers in the decision-making process, identify and describe cause-and-effect relationships between the main concepts of the subject area, and visualize possible situations. The paper describes the components of the ontological space of knowledge for the implementation of joint energy and socioecological studies of the impact of energy on the environment and quality of life. Examples of an ontology, a cognitive map, and a variant of the developed data model for designing a database for research are given.
\end{abstract}

Keywords: semantic modeling, cognitive modeling, ontology, ontological space, cognitive map, quality of life, decision support, database.

\section{References}

1. Avdeeva Z.K., Gorelova G.B., Kovriga S.V. Kognitivnye issledovaniya kachestva zhizni naseleniya goroda [Cognitive studies of the quality of life of the city population] // Trudy Sed'mogo Mezhdunarodnogo foruma po kognitivnomu modelirovaniyu. V 3-h chastyah: Trudy Tvorcheskoj shkoly. Trudy XH Mezhdunarodnoj konferencii. Trudy VII Mezhdunarodnoj konferencii $=$ Proceedings of the Seventh International forum on cognitive modeling. In 3 parts: 
Works of the Creative school. Works of the XX International conference, Works of the VII International conference. 2019. Pp. 205-214. (in Russian)

2. Avdeeva Z.K., Kovriga S.V., Makarenko D.I. Kognitivnoe modelirovanie dlya resheniya zadach upravleniya slabostrukturirovannymi sistemami (situaciyami) [Cognitive modeling for solving problems of managing weakly structured systems (situations)] // Upravlenie bol'shimi sistemami = Management of large systems. 2007. №16. Pp. 26-39. (in Russian)

3. Barker R. CASE*Method. Modelirovanie vzaimosvyazej mezhdu sushchnostyami [CASE*Method. The modeling of relationships between entities]. M.. 1992. 233 p. (in Russian) 4. Bol'shaya rossijskaya enciklopediya [Great Russian encyclopedia] Available at: https://bigenc.ru/vocabulary (accessed 8.10.2020). (in Russian)

5. Vorozhcova T.N. Ontologicheskaya model' prostranstva znanij dlya situacionnogo upravleniya $\mathrm{v}$ energetike [Ontological model of the knowledge space for situational management in the energy sector] // Trudy HKH Bajkal'skoj Vserossijskoj konferencii «Informacionnye i matematicheskie tekhnologii v nauke i upravlenii» = Proceedings of the XX Baikal all-Russian conference "Information and mathematical technologies in science and management». Irkutsk: ISEM SO RAN. 2015. Vol.3. Pp. 85-88. (in Russian)

6. Vorozhcova T.N., Makagonova N.N., Massel' L.V. Ontologicheskij podhod k proektirovaniju bazy dannyh dlja ocenki vlijanija jenergetiki na okruzhajushhuju sredu [Ontological approach to the design of a database for assessing the impact of energy on the environment] // «Informacionnye i matematicheskie tekhnologii $\mathrm{v}$ nauke i upravlenii»= Information and mathematical technologies in science and management. 2019. № 3 (15). Pp. 31-41. DOI: 10.25729/2413-0133-2019-3-03. (in Russian)

7. Gavrilova T. A., Kudryavcev D. V., Muromcev D. I. Inzheneriya znanij. Modeli i metody [Knowledge engineering. Models and methods]. Sankt-Peterburg. 2016. 324p. (in Russian)

8. Deyt K. Dzh. Vvedeniye v sistemy baz dannykh [An Introduction to Database Systems] (8th Edition). Izdatel'skiy dom "Vil'yams = M .: Williams Publishing House. 2005. 1328 p.

9. Zagorul'ko YU. A., Borovikova O. I., Zagorul'ko G. B. Rol' Ontologii v Tekhnologii Postroeniya Tematicheskih Nauchnyh Internet-resursov [The role of Ontology In the technology Of building Thematic Scientific Internet resources] // Vserossijskaya konferenciya $s$ mezhdunarodnym uchastiem "Znaniya - Ontologii - Teorii" (ZONT-2015) = All-Russian conference with international participation "Knowledge-Ontologies-Theories". Novosibirsk. Institut matematiki im. S.L. Soboleva SO RAN. 2015. Pp. 101- 110 (in Russian)

10. Zagorul'ko YU. A., Zagorul'ko G. B. Ontologii i ih prakticheskoe primenenie v sistemah, osnovannyh na znaniyah [Ontologies and their practical application in knowledge-based systems] // Vserossijskaya konferenciya s mezhdunarodnym uchastiem "Znaniya - Ontologii Teorii" (ZONT-2011) = All-Russian conference with international participation "KnowledgeOntologies-Theories". 2011. Vol. 1. (in Russian)

11. Kogalovskij M.R., Kalinichenko L.A. Konceptual'noe i ontologicheskoe modelirovanie v informacionnyh sistemah [Conceptual and ontological modeling in information systems] // Programmirovanie $=$ Programming. 2009. V. 35. № 5. Pp. 3-25 (in Russian).

12. Kuznecov O.P., Kulinich A.A., Markovskij A.V. Analiz vliyanij pri upravlenii slabostrukturirovannymi situaciyami na osnove kognitivnyh kart [Analysis of influences in the management of semi-structured situations based on cognitive maps] // CHelovecheskij faktor v upravlenii = Human factor in management. M.: KomKniga. 2006. Pp. 313-344. (in Russian) 
13. Kulinich A.A. Semioticheskie kognitivnye karty. CH. 1. Kognitivnyj i semioticheskij podhody v informatike i upravlenii [Semiotic cognitive maps. Part 1. Cognitive and semiotic approaches in computer science and management] // Problemy upravleniya = Management problems. 2016. № 1. Pp. 2-10. (in Russian)

14. Massel' A.G. Kognitivnoe modelirovanie v issledovaniyah problem energeticheskoj bezopasnosti: primeneniya i perspektivy razvitiya. [Cognitive modeling in energy security research: applications and development prospects] // Trudy Mezhdunarodnogo Kongressa po intellektual'nym sistemam i informacionnym tekhnologiyam «IS\&IT' 14» = Proceedings of The international Congress on intelligent systems and information technologies "IS\&IT' 14». M.: Fizmatlit. 2014. V.2. Pp. 153-158. (in Russian)

15. Massel' L.V., Vorozhcova T.N., Kopajgorodskij A.N., Makagonova N.N., Skripkin S.K. Primenenie ontologij v issledovaniyah i podderzhke prinyatiya reshenij v energetike [Application of ontologies in research and decision support in the energy sector] // Vserossijskaya konferenciya s mezhdunarodnym uchastiem «Znaniya - Ontologii - Teorii» $(Z O N T-13)=$ AllRussian conference with international participation "Knowledge-Ontologies-Theories". Novosibirsk: IM SO RAN. 2013. V.2. Pp. 29-38. (in Russian)

16. Massel' L.V. Primenenie ontologicheskogo, kognitivnogo i sobytijnogo modelirovaniya dlya analiza razvitiya i posledstvij chrezvychajnyh situacij $\mathrm{v}$ energetike [The use of ontological, cognitive and event modeling to analyze the development and consequences of emergency situations in the energy sector] // Problemy bezopasnosti i chrezvychajnyh situacij = Security and emergency issues. 2010. № 2. Pp.34-43. (in Russian)

17. Massel' L. V. Fraktal'nyj podhod k strukturirovaniyu znanij i primery ego primeneniya [Fractal approach to knowledge structuring and examples of its application] // Ontologiya proektirovaniya = Ontology of design. 2016. №2 (20). Pp. 149-161. (in Russian)

18. Tuzovskij A.F., Chirikov S.V., Jampol'skij V.Z. Sistemy upravlenija znanijami (metody i tehnologii) [Knowledge management systems (methods and technologies)]. Tomsk: NTL publ. 2005. 260 p. (in Russian)

19. CHernikov B.V. Leksikologicheskij sintez dokumentov v kompleksah informacionnyh sistem [Lexicological synthesis of documents in information systems complexes] // M: «Forum». 2017. 336 p. (in Russian)

20. Axelrod R. The Structure of Decision: Cognitive Maps of Political Elites. Princeton. University Press. 1976

21. Gruber T.R., Towards principles for the design of ontologies used for knowledge sharing // International Journal of Human-Computer Studies. 1995. V. 43. (5/6). 907 - 928.

22. Groumpos P., Stylios C., Modelling supervisory control systems using fuzzy cognitive maps, Chaos, Solitons \& Fractals. 2000. Vol. 11(1-3). 329-336.

23. Guarino N., Formal Ontology and Information Systems // Proceedings of International Conference of Formal Ontology and Information Systems (FOIS'98). Amsterdam: IOS Press. 1998/ Pp. $3-15$ 